Note

\title{
Development of a routine procedure for single nucleotide polymorphism marker design based on the $T_{m}$-shift genotyping method
}

\author{
Hiroyuki Fukuoka*, Koji Miyatake, Satomi Negoro, Tsukasa Nunome, Akio Ohyama \\ and Hirotaka Yamaguchi
}

Molecular Genetics and Physiology Research Team, National Institute of Vegetable and Tea Science (NIVTS), 360 Kusawa, Ano, Tsu, Mie 514-2392, Japan

\begin{abstract}
To establish a routine procedure for SNP marker development, the previously reported $\mathrm{T}_{\mathrm{m}}$-shift SNP genotyping procedure was validated and extensively improved. The effect of introducing mismatched nucleotides into allele-specific primers, using alternative fluorescent dyes, and varying the DNA polymerase species on SNP discrimination was examined at more than 100 known SNP loci in a solanaceous crop, eggplant. As a result, it has been shown that a success rate over $80 \%$ can be achieved with $\mathrm{T}_{\mathrm{m}}$-shift SNP genotyping using directional trials with automatically designed primer sets and a routine protocol for the reaction condition tests.
\end{abstract}

Key Words: genetic marker, SNPs, primer design, $\mathrm{T}_{\mathrm{m}}$-shift primer, single-tube genotyping, melting curve analysis.

A number of techniques for SNP genotyping have been reported, including those based on allele-specific PCR (Sommer et al. 1992), single-base extension (Syvanen 2001), probe hybridization (Livak et al. 1995, Piatek et al. 1998, Schutz et al. 2000), and microarray technology (Hirschhorn et al. 2000), among others (Kwok 2001). Each method has advantages and disadvantages compared with the others in regard to cost, specificity, and throughput, and no clearly superior method has been established. Among them, $\mathrm{T}_{\mathrm{m}}$-shift SNP genotyping (Germer and Higuchi 1999, Wang et al. 2005), a method that combines allele-specific PCR with discrimination of PCR products by melting-curve analysis, has the advantages that it does not require fluorescently labeled primers and that alleles are identified without any additional reactions or experimental manipulation; therefore, this typing method is likely to be quite useful when a relatively large number of SNPs are to be genotyped in a short period of time at reasonable cost. Versatility and compatibility with every SNP of interest, however, is one of the most important features of the SNP typing method when a large number of SNP markers are to be developed. Here we report the validation of the previously reported $\mathrm{T}_{\mathrm{m}}$-shift SNP genotyping procedure and improvements to the marker development process to establish a routine procedure for SNP marker development.

Communicated by T. Yamamoto

Received June 16, 2008. Accepted September 3, 2008.

*Corresponding author (e-mail: h.fukuoka@affrc.go.jp)
Optimization of reaction conditions and primer design to improve allele discrimination

All experiments in this study were performed using a solanaceous crop species, eggplant (Solanum melongena L.). Preliminary evaluation experiments were conducted following the procedure described by Wang et al. (2005) completely except that, because the heat-activated Stoffel-Gold enzyme was commercially unavailable, the standard Stoffel fragment (Applied Biosystems, Foster City, CA) was used with anti-Taq antibody (Platinum Taq Antibody, Invitrogen, Carlsbad, CA) to enable the hot-start reaction, as recommended by the authors. An example of the SNP locus examined in the trial experiment is shown in Fig. 1. Although Wang et al. (2005) reported a success rate of more than $80 \%$, our SNP genotyping was successful for only 1 SNP site (C/A allele) of the 5 sites tested (two $\mathrm{C} / \mathrm{T}$, two $\mathrm{C} / \mathrm{A}$, and one $\mathrm{G} / \mathrm{T}$ alleles). It was strongly suggested, therefore, that some modifications should be made to the original procedure in order to improve $\mathrm{T}_{\mathrm{m}}$-Shift PCR genotyping as a standard method for SNP marker development.

In the 4 failed experiments, it seemed that allele-specific primers (ASPs) for $\mathrm{G}$ or $\mathrm{C}$ alleles tended also to work on $\mathrm{A}$ or $\mathrm{T}$ alleles with considerable efficiency, which led to low allele specificity. As an example, Fig. 2A shows the result of $\mathrm{T}_{\mathrm{m}}$-shift typing using pASP1 and pASP2, shown in Fig. 1. The low success rate observed in the preliminary experiments was not likely to be due to the peculiar nucleotide sequences neighboring the SNP sites since no specific structures existed, such as long homopolymers, highly GC/AT rich regions or simple sequence repeats. Another possibility 


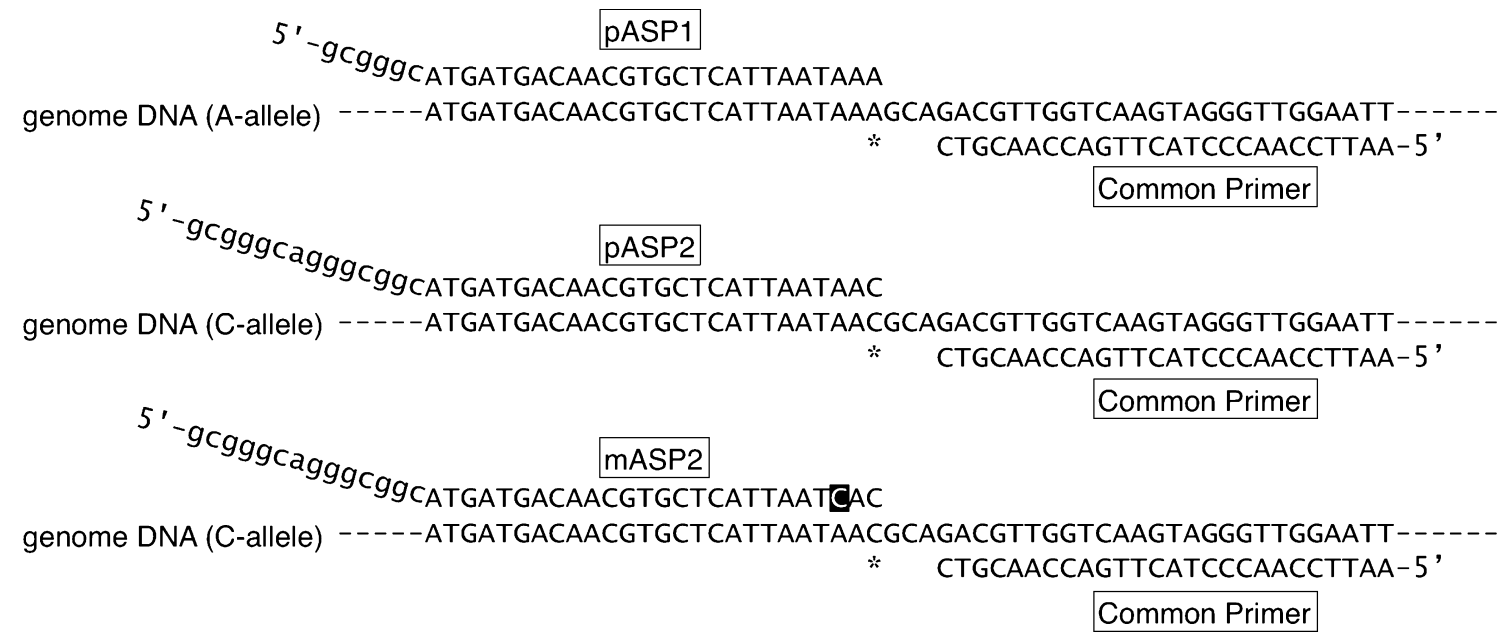

Fig. 1. Example of primer design for $\mathrm{T}_{\mathrm{m}}$-shift PCR genotyping. Genomic DNA sequences and primers corresponded to the data shown in Fig. 2A and 2B. ASP1 and ASP2 are allele-specific primers (ASPs) for A allele and C allele, respectively. Lower-case letters at the 5'-end of ASPs indicate short (pASP1) and long (pASP2 and mASP2) GC-rich tails. Asterisks indicate SNP positions. A mismatch nucleotide (C) introduced in mASP is shown as a white letter on a black background.

is that the lower success rate was owing to the use of the standard (non-heat-activated) version of the Stoffel fragment polymerase. The original report remarked that greater care should be taken in primer design to eliminate mispriming and dimerization of the primers when the standard Stoffel fragment was used. No practical procedures or methods were provided except for the anti-Taq antibody to suppress undesired priming, the effect of which seemed to be insufficient in our data.

In order to achieve higher allele specificity, an $\mathrm{A} / \mathrm{C}$ or $\mathrm{T} / \mathrm{G}$ mismatch was introduced at the $3 \mathrm{rd}$ nucleotide position from the 3 '-end of the ASPs (shown as mASP2 in Fig. 1), according to the method of Hayashi et al. (2004). The introduction of mismatched nucleotides into the ASPs for both wild and mutant alleles did not improve allele specificity in any of the 4 SNPs (data not shown). In contrast, introduction of a mismatch into only the ASPs corresponding to $\mathrm{G} / \mathrm{C}$ alleles significantly enhanced allele discrimination (Fig. 2B), and 3 SNPs were successfully genotyped of the 4 loci that were unable to be typed with the original method. When this modification was adopted, both the Stoffel fragment and the native Taq polymerase produced acceptable results even without the anti-Taq antibody. It was also found that switching the fluorescent dye used for melting-curve analysis improved the results in some cases. The last SNP locus $(\mathrm{C} / \mathrm{T}$ allele) that failed in previous trials was finally successfully genotyped when a saturating double-stranded DNA staining dye, EvaGreen (Biotium, Inc., Hayward, CA), was used instead of a non-saturating dye, SYBR Green I (Fig. 2C, 2D). In addition, it was observed that for some primer sets, the reactions produced less stable results when EvaGreen was added to the reaction before PCR than when it was mixed in after PCR, just prior to melting-curve analysis (data not shown). This difference was not observed in the experiments using SYBR Green I.
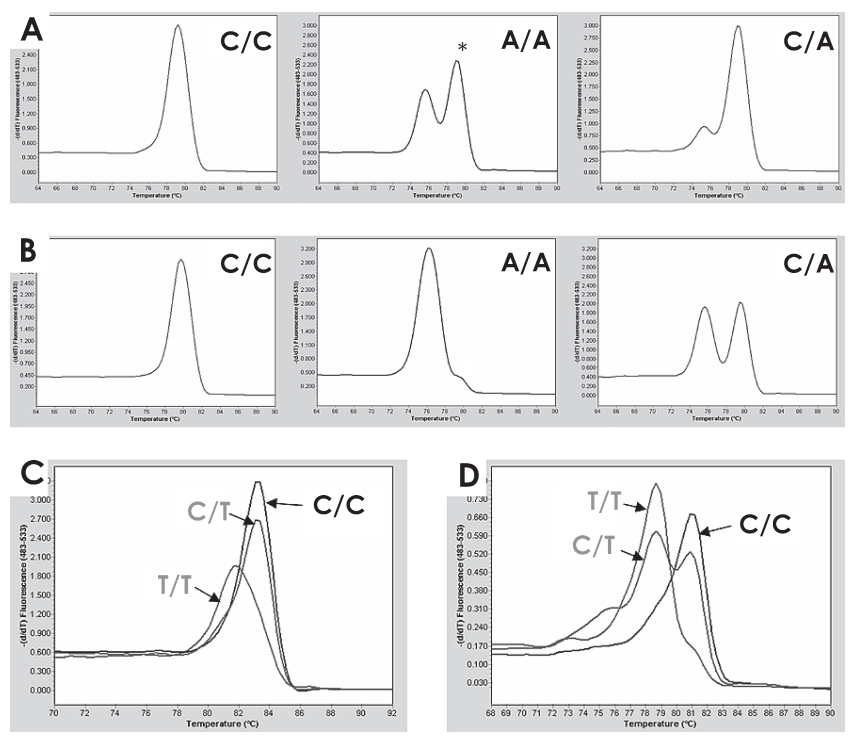

Fig. 2. Improved genotype discrimination of $\mathrm{T}_{\mathrm{m}}$-shift PCR genotyping. We tested the effect of introducing mismatches into allele-specific primers by genotyping a C/A SNP locus using a perfect-match primer set (A) or a mismatch primer set (B). The $3^{\prime}$-end sequences of allelespecific primers for the $\mathrm{C}$ allele and A allele were $5^{\prime}$-...ATAAC- $3^{\prime}$ and $5^{\prime}$-...ATAAA-3', respectively. In the mismatch primer set, the third nucleotide from the $3^{\prime}$-end of an allele-specific primer, A, was replaced with $\mathrm{C}$ to make mismatch primer $5^{\prime}-\ldots$ AT $\underline{\mathrm{C}} \mathrm{AC}-3^{\prime}$ only for the $\mathrm{C}$ allele-specific primer. Asterisks indicate false peaks derived from the $\mathrm{C}$ allele-specific primer in the A homozygote. We also examined the effect of different dyes. The result of C/T SNP discrimination using SYBR-Green I (C) and EvaGreen (D) is shown as an example.

Development of a tool for automatic primer design and establishment of a routine procedure for marker evaluation

Given the preliminary results shown above, we tried to develop SNP markers on a larger scale. In all experiments, 
PCR reactions were performed using standard PCR equipment (GeneAmp 9700 dual 384-well blocks, Applied Biosystems, Foster City, CA). After the reaction, the reaction plates were transferred to real-time PCR apparatus, LightCycler 480 (Roche Diagnostics, Indianapolis, IN), only for melting-curve analysis. The PCR reactions were set up as shown in Table 1. Two polymerase-dye combinations, native Taq polymerase plus SYBR Green I and Stoffel fragment plus EvaGreen, were compared, because the other combinations (Taq plus EvaGreen and Stoffel plus SYBR Green) exhibited no advantages or very little over the two combinations in the preliminary trials (data not shown). SYBR Green I was mixed in prior to the PCR reaction and EvaGreen was added after PCR and prior to melting-curve analysis based on the result of preliminary trials.

Perfect-match ASPs (pASPs) were designed for all alleles of $\mathrm{A} / \mathrm{C}, \mathrm{A} / \mathrm{G}, \mathrm{T} / \mathrm{C}$, and $\mathrm{T} / \mathrm{G}$ SNPs, whereas mismatch ASPs (mASPs) were tried only for $\mathrm{G}$ or $\mathrm{C}$ alleles. Only pASPs were used for A/T and G/C SNPs. Wang et al. (2005) reported that ASPs and common primers should be 14-17 mers and 22-27 mers, respectively, and the distance between the primers should be less than 20 bases. Because ready-made primer design tools were not efficiently applicable for dealing with the atypical parameters required in $T_{m^{-}}$ shift primer design, we developed a simple Perl script, 'tms_primer_designer.pl' in order to design PCR primer sets for $\mathrm{T}_{\mathrm{m}}$-shift genotyping. In association with a standard primer design tool, Primer3 (Rozen and Skaletsky 2000), the script generates all possible primer pairs on both strands that meet the conditions described above and selects the best primer pair based on a primer-pair penalty value calculated by Primer3. The script and accompanying document are available from the authors on request. The thermal cycling program of the PCR reaction and the thermal conditions in melting-curve analysis were set according to Wang's original report.

$\mathrm{T}_{\mathrm{m}}$-shift PCR-based marker development was tested for 137 SNPs. All primer sets were designed automatically using 'tms_primer_designer.pl' without any further manual inspection. Four primer-enzyme-dye combinations were examined for all primer sets and the combination of TaqSYBR-mASP showed the highest success rate, 74\% (Table 2). Although the combination of Stoffel-EvaGreen-mASPs resulted in the lowest success rate $(43 \%)$, some SNPs could be discriminated only by this combination. Of the 137 SNPs, $117(85 \%)$ were successfully identified in at least one reaction condition, and only $15 \%$ were unable to be genotyped with any set of conditions used in the experiment. Because only one primer set was tested for each SNP (with and/or without a mismatch in the ASP) designed by 'tms_primer_designer.pl', some portion of the failed SNPs may be successfully typed using other primer pairs, for example, those designed on opposite strands. To establish a common procedure for developing $\mathrm{T}_{\mathrm{m}}$-shift PCR markers, 25 SNP loci were randomly selected and the success rate was simulated under 4 sets of experimental conditions run in
Table 1. Reaction components of two enzyme-dye combinations for $\mathrm{T}_{\mathrm{m}}$-shift PCR genotyping

\begin{tabular}{|c|c|c|c|}
\hline \multicolumn{2}{|c|}{ Taq-SYBR GreenI combination } & \multicolumn{2}{|c|}{ Stoffel-EvaGreen combination* } \\
\hline genome DNA & $10 \mathrm{ng}$ & genome DNA & $10 \mathrm{ng}$ \\
\hline Tris-HCl ph 8.3 & $10 \mathrm{mM}$ & Tris- $\mathrm{HCl}$ ph 8.3 & $10 \mathrm{mM}$ \\
\hline $\mathrm{MgCl}_{2}$ & $1.5 \mathrm{mM}$ & $\mathrm{MgCl}_{2}$ & $2 \mathrm{mM}$ \\
\hline $\mathrm{KCl}$ & $65 \mathrm{mM}$ & $\mathrm{KCl}$ & $40 \mathrm{mM}$ \\
\hline dNTP & $0.2 \mathrm{mM}$ each & dNTP & $0.2 \mathrm{mM}$ each \\
\hline DMSO & $2 \%$ & DMSO & $2 \%$ \\
\hline glycerol & $1.25 \%$ & glycerol & $1.25 \%$ \\
\hline SYBR Green I & $0.2 \times$ & primer & $0.2 \mu \mathrm{M}$ each \\
\hline primer & $0.2 \mu \mathrm{M}$ each & Stoffel fragment & $0.3 \mathrm{U}$ \\
\hline Taq polymerase & $0.3 \mathrm{U}$ & ddw & up to $10 \mu \mathrm{l}$ \\
\hline ddw & up to $10 \mu \mathrm{l}$ & $\begin{array}{l}* \text { after the PCR } \\
\text { of } 10 \mathrm{mM} \text { Tris- } \\
\text { EvaGreen, } 2 \% \mathrm{D}\end{array}$ & $\begin{array}{l}\text { eaction, add } 5 \mu \mathrm{l} \\
\mathrm{Cl} \mathrm{pH} 8.3,0.4 \times \\
\text { ASO }\end{array}$ \\
\hline
\end{tabular}

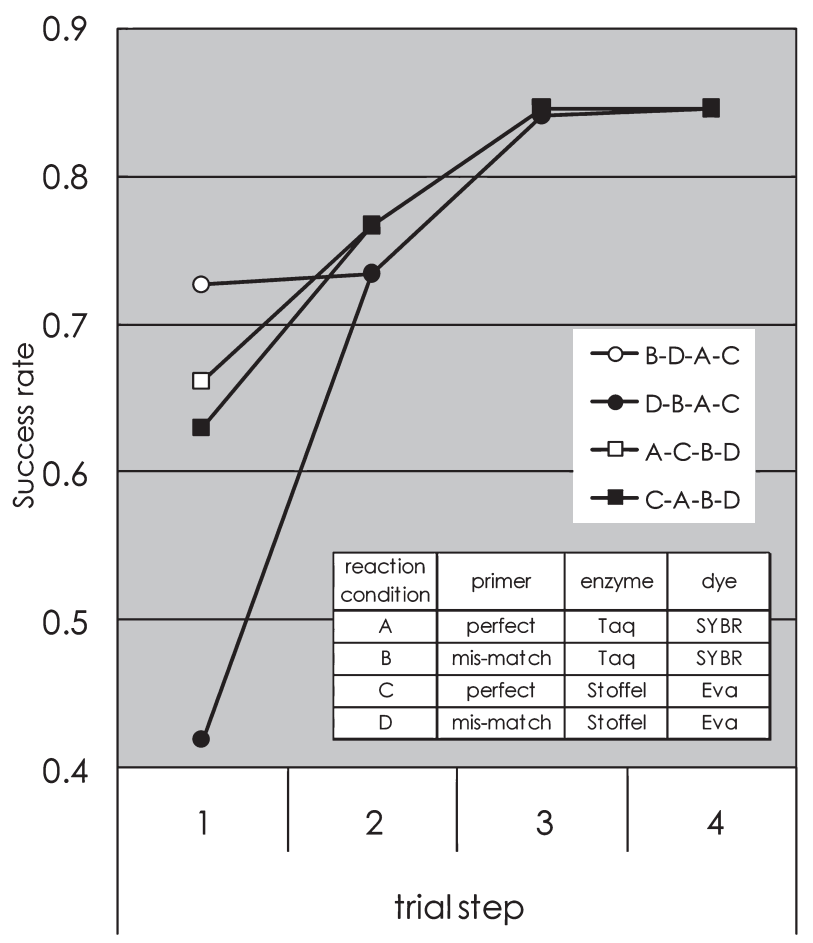

Fig. 3. Expected transition of success rate of $T_{m}$-shift PCR-based SNP marker development following trials with different experimental orders. The mean values of 50 simulations are plotted (see text). Each line legend (ex. B-D-A-C) indicates the order of the reaction conditions, as shown in the table in the inset.

different orders. The primer classes (i.e., pASP or mASP) for the first and second steps were set to be the same to minimize the cost of the second round synthesis of another class of primers. As shown in Fig. 3, even though the final success rate after the 4th trial step was the same in each order, the average of 50 replications of the simulation indicated that the best strategy to reduce the cost and effort of the experimental trials for practical marker development would be to first perform reactions with mASP, Taq polymerase and SYBR Green I, and then perfect-match primers should be 
Table 2. Success rate of $\mathrm{T}_{\mathrm{m}}$-shift PCR-based SNP marker development

\begin{tabular}{lcccccc}
\hline \hline \multirow{2}{*}{$\begin{array}{l}\text { C/G allele-specific primer } \\
\text { Polymerase Dye }\end{array}$} & & \multicolumn{2}{c}{ perfect match } & & \multicolumn{2}{c}{ mismatch } \\
\cline { 7 - 7 } \cline { 6 - 7 } & & $\begin{array}{c}\text { native Taq } \\
\text { SYBR Green I }\end{array}$ & $\begin{array}{c}\text { Stoffel fragment } \\
\text { EvaGreen }\end{array}$ & & $\begin{array}{c}\text { native Taq } \\
\text { SYBR Green I }\end{array}$ & $\begin{array}{c}\text { Stoffel fragment } \\
\text { EvaGreen }\end{array}$ \\
\hline total (137 loci) & & 0.65 & 0.64 & & 0.74 & 0.43 \\
randomly selected & mean & $0.66 \mathrm{ab}^{*}$ & $0.63 \mathrm{a}$ & & $0.73 \mathrm{~b}$ & $0.42 \mathrm{c}$ \\
25 loci (trials $=50)$ & min & 0.48 & 0.48 & & 0.6 & 0.28 \\
& max & 0.88 & 0.76 & & 0.84 & 0.6 \\
\hline
\end{tabular}

* Different characters indicate significant differences based on Kluskal-Wallis comparison $(p<0.01)$.

synthesized only for SNP loci that have not been successfully genotyped (order B-D-A-C). The second step (D) can be omitted because a quite limited number of SNP would be successful compared to the first step (B). In an experiment with the order A-C-B-D or C-A-B-D, however, the second trial step must be included because the success rate is significantly increased in the second step; therefore, it is suggested that order B-(D)-A-C is the best course to achieve the maximum success rate with the minimum experimental work and cost.

\section{Conclusion and Perspectives}

In this report, we have shown that a success rate over $80 \%$ can be achieved with $\mathrm{T}_{\mathrm{m}}$-shift SNP genotyping by using directional trials with automatically designed primer sets and a routine protocol for the reaction condition tests. The $\mathrm{T}_{\mathrm{m}}$-shift SNP markers developed in our experiments exhibited highly reproducible and clear results in linkage map construction experiments in eggplant and tomato (manuscript in preparation). These markers have also been applied for marker-assisted breeding programs where unrefined DNA samples prepared from a large number of individuals must be handled in a short period of time. Because it provides an improved and stabilized success rate for marker development, $\mathrm{T}_{\mathrm{m}}$-shift PCR will be an excellent method for SNP genotyping from the viewpoint of cost, reproducibility, ease, and versatility when there is a need to design and handle a relatively large number of markers.

\section{Acknowledgments}

This work was supported by the research program 'Development of DNA Marker-Aided Selection Technology for Plants and Animals' (DM-1608) and 'Development of DNA markers for expansion of genome research in the grass family into other crops' (DD-4010) funded by the Ministry of Agriculture, Forestry, and Fisheries of Japan.

\section{Literature Cited}

Germer, S. and R.Higuchi (1999) Single-tube genotyping without oligonucleotide probes. Genome Res. 9: 72-78.

Hayashi,K., N.Hashimoto, M.Daigena and I.Ashikawa (2004) Development of PCR-based SNP markers for rice blast resistance genes at the Piz locus. Theor. Appl. Genet. 208: 1212-1220.

Hirschhorn, J.N., P. Sklar, K. Lindblad-Toh, Y.M. Lim, M. RuizGutierrez, S.Bolks, B.Langhorst, S.Schaffner, E.Winchester and E.S.Lander (2000) SBE-TAGS: an array-based method for efficient single-nucleotide polymorphism genotyping. Proc. Natl. Acad. Sci. USA 12164-12169.

Kwok,P.-Y. (2001) Methods for genotyping single nucleotide polymorphisms. Ann. Rev. Genomics Hum. Genet. 2: 235-258.

Liviak,K.J., J.Marmaro and J.A.Todd (1995) Towards fully automated genome-wide polymorphism screening. Nat. Genet. 9: 341-342.

Piatek,A.S., S.Tyagi, A.C.Pol, A.Telenti, L.P.Miller, F.R.Kramer and D.Allend (1998) Molecular beacon sequence analysis for detecting drug resistance in Mycobacterium tuberculosis. Nat. Biotechnol. 16: 359-363.

Rozen,S. and H.J.Skaletsky (2000) Primer3 on the WWW for general users and for biologist programmers. In: Krawetz,S. and S.Misener (eds.) Bioinformatics Methods and Protocols: Methods in Molecular Biology. Humana Press, Totowa, NJ, pp. 365-386.

Schutz,E., N.von Ahsen and M.Oellerich (2000) Genotyping of eight thiopurine methyltransferase mutations: three-color multiplexing, 'two-color/shared' anchor and fluorescence-quenching hybridization probe assays based on thermodynamic nearest-neighbor probe design. Clin. Chem. 46: 1728-1737.

Sommer,S.S., A.R.Groszbach and C.D.Bottema (1992) PCR amplification of specific alleles (PASA) is a general method for rapidly detecting known single-base changes. Biotechniques 12: 82-87.

Syvanen,A.C. (2001) From gels to chips: "minisequencing" primer extension for analysis of point mutations and single nucleotide polymorphisms. Hum. Mutat. 13: 1-10.

Wang, J., K. Chuang, M. Ahluwalia, S. Patel, N.Umblas, D. Mirel, R.Higuchi and S.Germer (2005) High-throughput SNP genotyping by single-tube PCR with $\mathrm{T}_{\mathrm{m}}$-shift primers. BioTechniques 39: 885893. 